\title{
A COUNTEREXAMPLE IN DIFFERENTIAL ALGEBRA ${ }^{1}$
}

\author{
GEORGE M. BERGMAN
}

Kolchin [1, p. 791] calls a differential field extension "weakly normal" if the fixed field of its Galois group is the base field, and "nor$\mathrm{mal}^{\text {" }}$ if it is weakly normal over every subextension. He gives an example to show that if the extension is allowed to have a larger field of constants than the base field, these conditions are not equivalent, but he does not know whether they are equivalent for extensions preserving constants. The example in $\$ 1$ of the present paper shows that they are not. In $\$ 2$, we describe precisely the Galois group of this example.

I would like to express my indebtedness to Professor A. Seidenberg, whose fascinating course in differential algebra here at Harvard has been my introduction to the field.

1. The example. Let $A$ be a nontrivial additive subgroup of the complexes, $\boldsymbol{C}$, with a finite additive basis, $c_{1}, \cdots, c_{n}$; and let $G$ be the multiplicative group of those complex numbers $u$ such that $A$ is closed under multiplication by both $u$ and $u^{-1}$. Suppose that $G$ does not consist entirely of roots of unity. ${ }^{2}$

Let $F$ be the field of meromorphic functions generated over $C$ by $e^{z}$ and $e^{c 1 e^{z}}, \cdots, e^{c n e^{z}}$. These generators are easily shown to be algebraically independent.

The field is closed under differentiation. The differential equations satisfied by our generators are:

$$
\frac{d}{d z} e^{s}=e^{s}, \quad \frac{d}{d z} e^{c_{0} s}=c_{i} e^{z} e^{c_{i s} z} .
$$

Received by the editors April 13, 1964.

1 This work was done while the author held a National Science Foundation first year graduate fellowship.

2 For example, let $R$ be any finite integral algebraic extension ring of the integers, and $A$ any finitely generated $R$-submodule of $C$ (such as $R$ itself). $G$ will contain all the units of $R$. From the Dirichlet Unit Theorem, we know of a very large class of such rings $R$ having units other than roots of unity.

Conversely, given any $A$, if we take $R$ to be the ring of all complex numbers $u$ such that $u A \subset A$, then $G$ will consist exactly of the units of $R$. This $R$ must be of finite rank as an additive group (for $A$ is, and given any $a \neq 0$ in $A, R \subset(1 / a) A$ ), hence must be a finite integral extension of the integers.

As a concrete example, $G=\left\{ \pm(1+\sqrt{ } 2)^{n}\right\}_{n-\cdots-1,0.1,2 \cdots,}$ if $A$ is generated by 1 and $\sqrt{ } 2$. 
Now for any nonzero complex number $u$, consider the algebraic isomorphism $\sigma_{u}$ of $F$ defined by

$$
\begin{gathered}
e^{z} \rightarrow u e^{z}, \\
e^{c i e^{z}} \rightarrow e^{u c i e^{z}} .
\end{gathered}
$$

That it is a differential isomorphism can be checked either by verifying that the images of the generators satisfy (1), or by noting that it is the restriction to $F$ of the "translation"-automorphism $f(z)$ $\rightarrow f(z+\lambda)$ of the field of all meromorphic functions, where $\lambda$ is any logarithm of $u$.

If $u$ is not a root of unity, the only elements of $F$ invariant under this map are the constants. For suppose $f(z)$ is invariant. By the nature of the field $F, f(z)$ is a meromorphic function of $e^{z}: f(z)=m\left(e^{z}\right)$. Then we must have the identity $m(u \zeta)=m(\zeta)$. Expanding $m$ about zero in powers of $\zeta$, we find that it must consist of a constant term only.

If $u$ is a member of $G, \sigma_{u}$ will be an automorphism of $F$ over C. Taking $u$ to be a member of $G$ that is not a root of unity, we get an automorphism of $F$ over $\boldsymbol{C}$ whose fixed field is $\boldsymbol{C}$. So $F$ is weakly normal over $C$.

To show that it is not normal, we shall show that for some prime $p$, any automorphism $\sigma$ of $F$ over $C$ leaving $e^{p z}$ fixed leaves $e^{z}$ fixed as well, whence $F$ is not weakly normal over $\mathbf{C}\left(e^{p z}\right)$.

Let $\sigma$ be an arbitrary automorphism of $F$ over $C$. Since $\sigma\left(e^{z}\right)$ must satisfy the same differential equation as $e^{z}$, it must be of the form $u e^{z}$ for some $u \in C^{*}$ (the nonzero complexes). From this, we calculate in turn that for each $a \in A, \sigma\left(e^{a e^{*}}\right)$ must be of the form $k e^{a u \sigma^{*}}\left(k \in C^{*}\right.$, not necessarily the same for all $a$ ). The only elements of such a form in $F$ are the $k e^{a^{\prime} e^{z}}$ for $a^{\prime} \in A$, hence $a u=a^{\prime}$, hence multiplication by $u$ sends $A$ into itself. Looking at $\sigma^{-1}$, we similarly conclude that multiplication by $u^{-1}$ sends $A$ into itself. Hence $u \in G$. (This is not to say that $\sigma$ need equal $\sigma_{u}-$ cf. $\$ 2$.)

Now $G$ can contain at most finitely many roots of unity. (For it is the group of units of a finite algebraic extension of the integers-see footnote 2.) Hence for some prime $p$ it will not contain the primitive $p$ th roots of unity. Now suppose the $\sigma$ discussed above leaves $e^{p x}$ fixed. Then $e^{p x}=\sigma\left(e^{p z}\right)=\sigma\left(e^{z}\right)^{p}=u^{p} e^{p z}$, hence $u=1$, hence $\sigma$ leaves $e^{x}$ fixed. Q.E.D.

2. Further observations. From the argument begun above, we can see that any automorphism of $F$ must be of the form:

$$
\begin{aligned}
& e^{z} \rightarrow u e^{z} \quad(u \in G), \\
& e^{c i e^{z}} \rightarrow k_{i} e^{u 0 i e^{*}} \quad\left(k_{i} \in C^{*} ; i=1, \cdots, n\right) \text {. }
\end{aligned}
$$


We find that for all $u \in G$ and all values of $k_{1}, \cdots, k_{n}$ this gives an automorphism of $F$ (for the images of $e^{z}, e^{e_{i} e^{z}}$ are algebraically independent and satisfy the differential equations (1)). Let us designate this automorphism $\left(k_{1}, \cdots, k_{n}, u\right)$.

Let $X$ be any commutative group. The ring of $n \times n$ matrices with integral coefficients has an obvious action as a ring of endomorphisms on $X^{n}$-if $X$ is written multiplicatively, for instance, for any $n$-tuplet $x=\left(x_{1}, \cdots, x_{n}\right)$ of members of $X$ and any matrix $\bar{m}=\left(m_{i j}\right)$, we can write $x^{\bar{m}}=\left(\prod^{j} x_{j}^{m_{j j}}, \cdots, \prod^{j} x_{j}^{m_{j j}}\right)$. In particular, the group of invertible matrices acts as a group of automorphisms of $X^{n}$.

Given $u \in G$, let $\bar{u}$ be the matrix showing the action on $A$ of multiplication by $u$, in terms of the basis $c_{1}, \cdots, c_{n}$. Then we can check easily that our automorphisms compose by the rule $(k, u) \cdot\left(k^{\prime}, u^{\prime}\right)$ $=\left(k \cdot k^{\prime} \bar{u}, u u^{\prime}\right)$. Thus we have a semidirect product of $\left(C^{*}\right)^{n}$ and $G$.

\section{REFERENCE}

1. E. R. Kolchin, Galois theory of differential fields, Amer. J. Math. 75 (1953), 753-824.

Harvard UNIVERSITY 